\title{
Investigation of the effect of compression on a soft fibrous porous medium
}

\author{
S. Woudberg \\ Applied Mathematics Division, Department of Mathematical Sciences, \\ Stellenbosch University, Stellenbosch, South Africa
}

\begin{abstract}
An adaptable geometric pore-scale model is proposed to determine the effect of compression of a fibrous porous medium on the permeability. The model is applied to a soft compressible polyester material typically found in pillows. Seven stages of compression of the porous medium are considered. The effect of compression on the micro-structural parameters is determined, because it directly affects the permeability and pressure drop predictions. The model is also adapted to account for the combined effects of compression and developing flow on permeability. A sensitivity analysis is performed to determine the effect of experimental errors on the permeability prediction.

Keywords: permeability, porous media, compression, fluid dynamics, mathematical modelling, pressure drop, laminar, microstructure.
\end{abstract}

\section{Introduction}

According to Dukhan and Patel [1], compression of metallic foams increases the structural rigidity of the foam as well as its dynamic specific surface. This in turn leads to improved thermal performance of heat exchanger devices in which metallic foams find application [2]. Dukhan [3] also states that in the literature there often has to be relied on experimental correlations to investigate the influence of compression on permeability. This is due to the lack of geometrical foam models in the literature that can account for the effect of compression on permeability and inertial coefficients. Gerbaux et al. [2], Antohe et al. [4] and Boomsma and Poulikakos [5] also reverted to direct curve fitting of experimental results.

The aim of this study is to present a geometric model that can serve as a first attempt to fulfil this need in the literature. The model predictions for the 
permeability will be applied to available experimental data on the compression of a soft fibrous polyester material typically found in pillows. In Section 2 the adaptable geometric foam model will be presented. In Section 3 the model is applied to a soft fibrous porous medium. The influence of inertial effects and developing flow on the permeability are determined, followed by a sensitivity analysis in Section 3 and conclusions in Section 4.

\section{Characteristics of the anisotropic foam RUC model}

The existing transversely isotropic RUC (Representative Unit Cell) model of Du Plessis et al. [6] (shown in fig. 1(a)) is adapted geometrically to the anisotropic structure shown in fig. 1(b). The anisotropic model is obtained by shortening the strut in the streamwise direction. The streamwise direction also corresponds to the direction of compression.

The dimensions of the anisotropic RUC (as indicated in fig. 1(b)) in the plane perpendicular to the streamwise direction are $d_{\perp} \times d_{\perp}$, whereas in the streamwise direction the RUC extends over a distance $d_{\|}$(with $d_{\|}<d_{\perp}$ ). Although the struts may physically deform, it will be implicitly assumed that $d_{\|} \geq 2 d_{s}$, so that maximum compression excludes deformation of the struts from their original shape.

Let $U_{o}$ denote the total volume of the RUC, $U_{s}$ the total solid volume and $U_{f}$ the total fluid volume. It then follows that

$$
\begin{aligned}
U_{o} & =d_{\perp}^{2} d_{\|}, \\
U_{s} & =d_{s}^{2}\left(2 d_{\perp}-2 d_{s}+d_{\|}\right), \\
U_{f} & =d_{\perp}^{2} d_{\|}-d_{s}^{2}\left(2 d_{\perp}-2 d_{s}+d_{\|}\right) .
\end{aligned}
$$

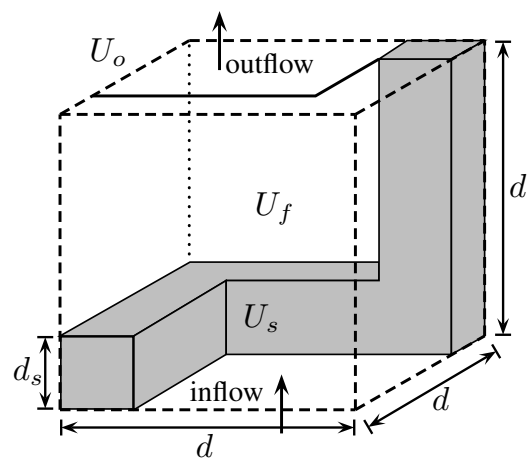

(a) Transversely isotropic model

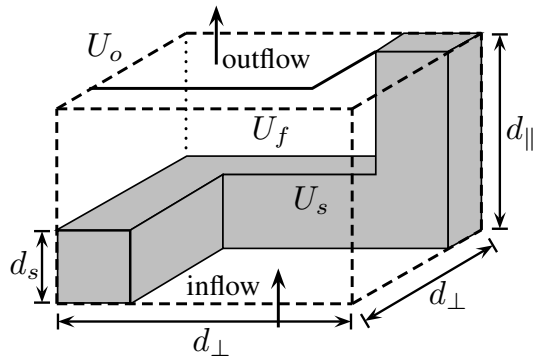

(b) Anisotropic model

Figure 1: (a) Transversely isotropic and (b) anisotropic foam RUC model. 
The porosity may, as a result, be expressed as

$$
\epsilon=\frac{U_{f}}{U_{o}}=\frac{d_{\perp}^{2} d_{\|}-d_{s}^{2}\left(2 d_{\perp}-2 d_{s}+d_{\|}\right)}{d_{\perp}^{2} d_{\|}} .
$$

\section{Application of the anisotropic foam model to soft fibrous media}

Akaydin et al. [7] provided experimental data for the permeability of airflow through soft fibrous porous media subject to compression. Polyester pillow material, consisting of fibres with an average diameter of $10 \mu \mathrm{m}$, was used. Each test sample of soft fibrous material consisted of several layers of randomly distributed fibres.

\subsection{Experimental results of Akaydin et al. [7]}

The experimental data are given in Table 1 for seven stages of compression.

In Table $1, h$ denotes the sample thickness in the direction of compression, $\mathrm{e}_{\exp }$ is the sample thickness relative to its uncompressed state and $C_{f}$ is the coefficient of the pressure gradient term in the Forchheimer flow regime, i.e.

$$
-\frac{\mathrm{d} p}{\mathrm{~d} x}=\frac{\mu q_{\exp }}{\epsilon k_{\exp }}+\frac{C_{f}}{\epsilon^{2}} \rho q_{\exp }^{2},
$$

with the $x$-direction corresponding to the direction of compression and $\mu$ and $\rho$ being the dynamic viscosity and density of air, respectively. The Reynolds number, $R e_{k}$, is defined as

$$
R e_{k}=\frac{\rho q_{\exp } \sqrt{k_{e x p}}}{\epsilon \mu} .
$$

Table 1: Experimental results for airflow through layers of soft polyester pillow material with average fibre diameter of $10 \mu \mathrm{m}$ [7].

\begin{tabular}{|c|c|c|c|c|c|c|}
\hline$h[\mathrm{~mm}]$ & $\mathrm{e}_{\exp }$ & $\epsilon$ & $k_{\exp }\left[10^{-9} \mathrm{~m}^{2}\right]$ & $C_{f}\left[10^{4} \mathrm{~m}^{-1}\right]$ & $R e_{k}$ & $q_{\exp }[\mathrm{m} / \mathrm{s}]$ \\
\hline 16 & 0.15 & 0.9780 & 0.62 & 2.58 & 0.07 & 0.0419 \\
30 & 0.27 & 0.9883 & 1.34 & 0.24 & 0.10 & 0.0412 \\
41 & 0.37 & 0.9914 & 2.11 & 0.19 & 0.13 & 0.0428 \\
50 & 0.45 & 0.9930 & 2.82 & 0.16 & 0.15 & 0.0428 \\
60 & 0.55 & 0.9941 & 3.72 & 0.21 & 0.17 & 0.0423 \\
86 & 0.78 & 0.9959 & 6.17 & 0.15 & 0.22 & 0.0426 \\
110 & 1.0 & 0.9968 & 9.22 & 0.08 & 0.26 & 0.0412 \\
\hline
\end{tabular}


An experimental error range of $4 \%$ in the $k_{e x p}$-values is reported by Akaydin et al. [7].

\subsection{Application of the anisotropic RUC model}

The width of the struts of the RUC model is set equal to the average diameter of the fibres, i.e. $d_{s}=10 \mu \mathrm{m}$. The effect of compression on both $d_{\|}$and $d_{\perp}$ has to be determined. Apart from knowing the value of $d_{s}$, more information on the micro-structural geometry is needed.

The difficulty, however, is that the measured values for the mean hydraulic pore diameter, at the different stages of compression, were not provided. As a result, the short strut length in the uncompressed state, $d_{\|_{o}}$, cannot be determined from the experimental mean hydraulic pore diameter values. Although not being an optimal approach, one has to rely on the predictions for the micro-structural parameters provided by the transversely isotropic RUC model to determine the value of $d_{\|_{o}}$.

\subsection{Calculation of micro-structural parameters}

The value of $d_{\|_{o}}$ is obtained from the relation between the pore-scale linear dimensions provided by the transversely isotropic RUC model (refer to fig. 1(b)), i.e.

$$
d_{\|_{o}}=\frac{2 d_{s}}{\psi_{o}-1},
$$

where $\psi_{o}$ denotes the geometric factor in the uncompressed state, i.e.

$$
\psi_{o}=2+2 \cos \left[\frac{4 \pi}{3}+\frac{1}{3} \cos ^{-1}\left(2 \epsilon_{o}-1\right)\right] .
$$

In eqn. (8), $\epsilon_{o}$ denotes the porosity of the uncompressed state, i.e. $\epsilon_{o}=0.9968$. The latter porosity value yields $d_{\|_{o}}=302.8 \mu \mathrm{m}$. An underlying assumption in the latter procedure of determining the value of $d_{\|_{o}}$ is that $d_{\|}=d_{\perp}$ for $\epsilon_{o}=0.9968$, which is not necessarily the case, since even in the uncompressed state it may be that $d_{\|}<d_{\perp}$. The value of $d_{\|_{o}}=302.8 \mu \mathrm{m}$ should thus be regarded as a first order approximation.

The dependence of the sample thickness relative to its uncompressed state, i.e. $\mathrm{e}_{\text {exp }}$, on porosity is not linear. To find an equation that fits the experimental data for $\mathrm{e}_{\exp }$ as a function of porosity the power addition technique of Churchill and Usagi [8] is applied to the asymptotes passing through the first and last two data points, yielding

$$
\mathrm{e}=\left[\left(8.66 \times 10^{-26} e^{57.1 \epsilon}\right)^{s}+\left(3.08 \times 10^{-120} e^{276.1 \epsilon}\right)^{s}\right]^{1 / s},
$$

where $s$ is the shifting exponent. The RUC thickness in the streamwise direction, $d_{\|}$, can be obtained by assuming that

$$
d_{\|}=d_{\|_{o}} \mathrm{e} .
$$


Knowing $d_{\|}$, the transverse RUC dimension, $d_{\perp}$, can be obtained from eqn. (4), yielding

$$
d_{\perp}=\frac{d_{s}\left(d_{s}+\sqrt{d_{s}^{2}+(1-\epsilon) d_{\|}\left(d_{\|}-2 d_{s}\right)}\right)}{(1-\epsilon) d_{\|}} .
$$

\subsection{Permeability prediction}

The relationship between the magnitudes of the streamwise average channel velocity, $w_{\|}$, and the superficial velocity, $q$, follows from mass conservation and is given by

$$
w_{\|}=\frac{q \psi}{\epsilon}=\frac{q d_{\perp}^{2}}{\left(d_{\perp}-d_{s}\right)^{2}},
$$

where the geometric factor $\psi$, which in this case is equal to the geometrical tortuosity, is given by

$$
\psi=\frac{\epsilon d_{\perp}^{2}}{\left(d_{\perp}-d_{s}\right)^{2}} .
$$

The wall shear stress for plane-Poiseuille flow in the streamwise channel can be expressed as

$$
\tau_{w_{\|}}=\frac{6 \mu w_{\|}}{\left(d_{\perp}-d_{s}\right)},
$$

and for the two transverse channels

$$
\tau_{w_{\perp_{1}}}=\frac{6 \mu w_{\perp}}{\left(d_{\|}-d_{s}\right)} \quad \text { and } \quad \tau_{w_{\perp_{2}}}=\frac{6 \mu w_{\perp}}{\left(d_{\perp}-d_{s}\right)} .
$$

The total pressure drop over the RUC, $\Delta p$, in the limit of low Reynolds number flow may be expressed as

$$
\Delta p=\frac{S_{\|} \tau_{w_{\|}}}{A_{p_{\|}}}+\frac{\xi S_{\perp} \tau_{w_{\perp}}}{A_{p_{\|}}} .
$$

From eqns. (12) to (16), it follows that

$$
\Delta p=\frac{24 d_{s} d_{\perp}^{2} \mu q}{\left(d_{\perp}-d_{s}\right)^{2}}\left[\frac{1}{A_{p_{\|}}}+\frac{\xi\left(d_{\perp}-d_{s}\right)}{A_{p_{\perp}}\left(d_{\|}-d_{s}\right)}+\frac{\xi\left(d_{\|}-d_{s}\right)}{A_{p_{\perp}}\left(d_{\perp}-d_{s}\right)}\right] .
$$

The pressure drop over the streamwise extent, $d_{\|}$, of the RUC is assumed to be the same as the pressure gradient over the fibrous porous medium, yielding

$$
\frac{\Delta p}{d_{\|}}=\frac{24 d_{s} d_{\perp}^{2} \mu q}{d_{\|}\left(d_{\perp}-d_{s}\right)^{2}}\left[\frac{2}{\left(d_{\perp}-d_{s}\right)^{2}}+\frac{1}{\left(d_{\|}-d_{s}\right)^{2}}\right],
$$

where the permeability is given by

$$
k=\frac{d_{\|}\left(d_{\perp}-d_{s}\right)^{2}}{24 d_{s} d_{\perp}^{2}}\left[\frac{2}{\left(d_{\perp}-d_{s}\right)^{2}}+\frac{1}{\left(d_{\|}-d_{s}\right)^{2}}\right]^{-1} .
$$




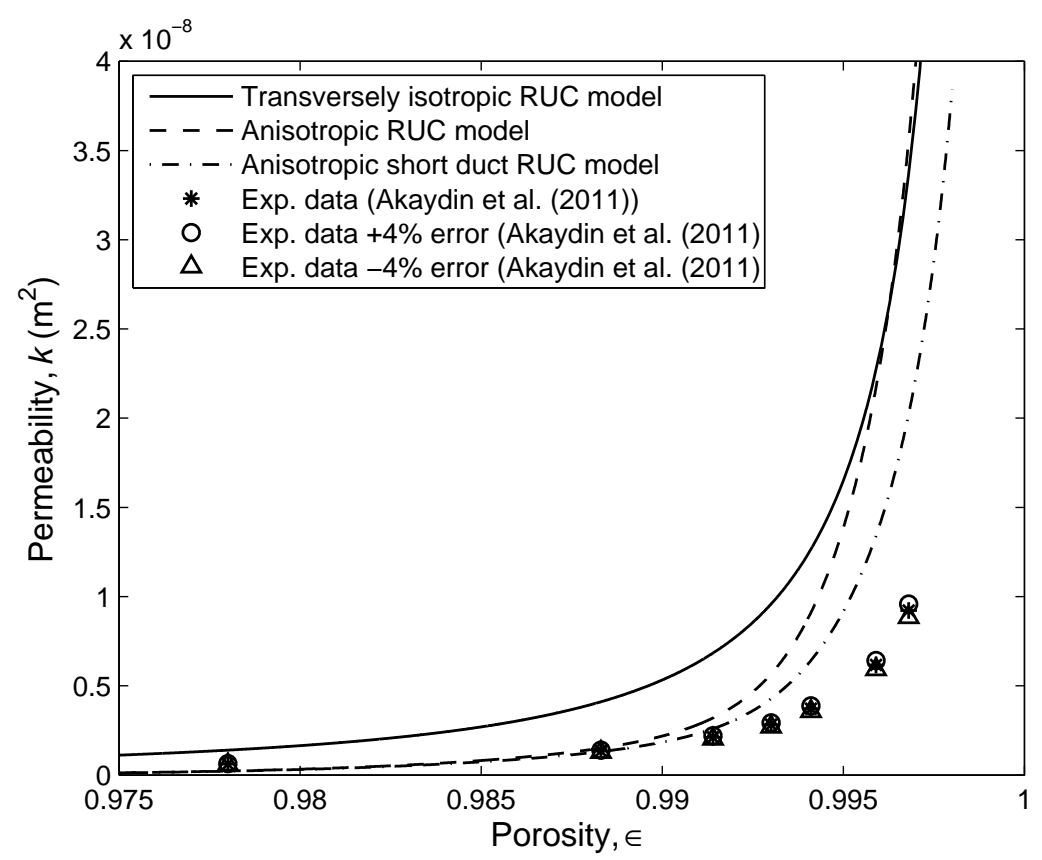

Figure 2: Permeability of compressible soft fibrous media predicted by the isotropic, anisotropic and anisotropic short duct RUC models.

Fig. 2 compares the permeability predicted by the anisotropic RUC model with the experimental permeability data of Akaydin et al. [7]. Also shown for comparison is the permeability predicted by the transversely isotropic RUC model and a $4 \%$ error in the experimental permeability data. The two model predictions yield the same values for no-compression (i.e. at $\epsilon=0.9968$ ), as expected.

From fig. 2 it is evident that compression has a significant effect on permeability. For the narrow porosity range of less than 0.02 the permeability changes with more than an order of magnitude. Both the transversely isotropic and anisotropic RUC models over-predict the experimental permeability values. The absolute relative percentage errors are given in Table 2. The anisotropic model decreases the over-prediction of the transversely isotropic model by an average of $94.0 \%$. The anisotropic model, however, still over-predicts the permeability. This overprediction tends to increase with an increase in porosity. Possible physical explanations for the over-prediction are investigated in the following subsection. 
Table 2: Absolute relative percentage error values for the permeability prediction of soft fibrous media.

\begin{tabular}{|c|c|c|c|c|}
\hline \multicolumn{2}{|c|}{} & \multicolumn{3}{|c|}{$\begin{array}{c}\text { Absolute relative percentage } \\
\text { error for } k(\%)\end{array}$} \\
\hline & & $\begin{array}{l}\text { Transversely } \\
\text { isotropic }\end{array}$ & Anisotropic & $\begin{array}{c}\text { Anisotropic } \\
\text { short duct }\end{array}$ \\
\hline 0.15 & 0.9780 & 125.0 & 63.7 & 65.1 \\
0.27 & 0.9883 & 206.2 & 12.5 & 2.0 \\
0.37 & 0.9914 & 224.2 & 51.8 & 23.0 \\
0.45 & 0.9930 & 239.8 & 100.9 & 51.6 \\
0.55 & 0.9941 & 240.1 & 144.6 & 72.5 \\
0.78 & 0.9959 & 268.2 & 252.3 & 117.5 \\
1.0 & 0.9968 & 265.6 & 285.8 & 119.5 \\
\hline
\end{tabular}

\subsection{Determining the influence of inertial effects}

The values for $q_{\exp }$ are presented in Table 1 . The average of these $q_{\text {exp }}$-values is $q_{\text {exp }}=0.042 \mathrm{~m} / \mathrm{s}$. For Reynolds numbers, defined in terms of the hydraulic diameter, as

$$
R e_{D h}=\frac{\rho w_{\|} D_{h}}{\mu}=\frac{\rho q_{\exp } d_{\perp}^{2} D_{h}}{\mu\left(d_{\perp}-d_{s}\right)^{2}},
$$

values in the order of $10^{-1}$ are obtained. The inertial forces are therefore negligible in comparison with the viscous forces. Next, the effect of developing flow on permeability is investigated.

\subsection{Determining the influence of developing flow}

In order to account for the effect of developing flow on permeability the streamwise pressure gradient may be expressed as

$$
\begin{aligned}
\frac{\Delta p}{d_{\|}} & =f_{a p p} \frac{1}{2} \rho w_{\|}^{2} \frac{4}{D_{h}}+f_{a p p} \frac{1}{2} \rho w_{\perp}^{2} \frac{4}{D_{h}} \\
& =\frac{d_{\perp}^{2} \mu q_{e x p}}{2 \psi d_{\|}\left(d_{\perp}-d_{s}\right)^{2}}\left[f_{a p p} R e_{D h} \frac{S_{\|}}{A_{p_{\|}} D_{h}}+f_{a p p} R e_{D h} \frac{S_{\perp}}{A_{p_{\perp}} D_{h}}\right]
\end{aligned}
$$

All the values for $\psi$ predicted by the anisotropic RUC model are close to unity. It was therefore assumed that $\psi=1$. The apparent friction factor-Reynolds number 
product is given by Shah and London [9] as

$$
f_{\text {app }} R e_{D h}=12 \sqrt{1+\frac{0.0822 D_{h} R e_{D h}}{d_{s}}} .
$$

The streamwise pressure gradient may consequently be expressed as

$$
\begin{aligned}
& \frac{\Delta p}{d_{\|}}=\frac{24 d_{\perp}^{2} \mu q_{\exp }}{d_{\|}\left(d_{\perp}-d_{s}\right)^{2}} {\left[2 \sqrt{1+\frac{0.0822 \rho q_{\exp } d_{\perp}^{2}}{\mu d_{s}}} \frac{d_{s}}{\left(d_{\perp}-d_{s}\right)^{2}}\right.} \\
&\left.+\sqrt{1+\frac{0.0822\left(d_{\|}-d_{s}\right)^{2} \rho q_{\exp } d_{\perp}^{2}}{\mu d_{s}\left(d_{\perp}-d_{s}\right)^{2}}} \frac{d_{s}}{\left(d_{\|}-d_{s}\right)^{2}}\right]
\end{aligned}
$$

The permeability predicted by the anisotropic short duct RUC model is then given by

$$
\begin{aligned}
& k=\frac{d_{\|}\left(d_{\perp}-d_{s}\right)^{2}}{24 d_{\perp}^{2}} {\left[2 \sqrt{1+\frac{0.0822 \rho q_{\exp } d_{\perp}^{2}}{\mu d_{s}}} \frac{d_{s}}{\left(d_{\perp}-d_{s}\right)^{2}}\right.} \\
&\left.+\sqrt{1+\frac{0.0822\left(d_{\|}-d_{s}\right)^{2} \rho q_{\exp } d_{\perp}^{2}}{\mu d_{s}\left(d_{\perp}-d_{s}\right)^{2}}} \frac{d_{s}}{\left(d_{\|}-d_{s}\right)^{2}}\right]^{-1} .
\end{aligned}
$$

The permeability prediction of eqn. (24) is shown graphically in fig. 2. The absolute relative percentage errors are given in Table 2 .

The incorporation of the effect of developing flow into the modelling procedure of the anisotropic RUC model reduced the over-prediction of the anisotropic model significantly (i.e. with $65.7 \%$, on average). The absolute relative percentage errors, however, still range from $2.0 \%$ to $119.5 \%$. In the next section a sensitivity analysis is performed to determine the effect of error ranges in the input parameters of the model.

\section{Sensitivity analysis}

Although estimates for experimental error ranges in $\mathrm{e}_{\exp }$ are not provided by Akaydin et al. [7], an experimental error range of $5 \%$ was assumed for this parameter. For $\epsilon$ an appropriate experimental error range of $0.1 \%$ was used (ranges larger than $0.5 \%$ lead to values in $\epsilon$ larger than unity.). For $q_{\text {exp }}$ an experimental error range of $5 \%$ was chosen but it was found that this error range has a negligible effect on permeability. A change in $\mathrm{e}_{\exp }$ affects the expression for e, given by eqn. (9) and, consequently, the value of $d_{\|_{o}}$. A change in $\epsilon$ also affects the expression for e and the value of $d_{\|_{o}}$. It was found that the permeability prediction is very sensitive to deviations in the values of $d_{\|_{o}}$ and to deviations in the porosity values used in the model. The permeability prediction is affected to a much lesser degree by the compression ratio. 
The influence of a $50 \%, 25 \%$ and $5 \%$ error in $d_{\|_{o}}$, in combination with a $0.1 \%$ error in $\epsilon$ and a $5 \%$ error in $\mathrm{e}_{\text {exp }}$ were investigated. Starting with an error of $50 \%$ in $d_{\|_{o}}$, the combination of percentage errors that leads to the largest deviation in the permeability prediction are given by the following two conditions:

- Condition (i): $50 \%$ added to all the values of $d_{\|_{o}} ; 5 \%$ added to all the values of $\mathrm{e}_{\text {exp }}$ and $0.1 \%$ subtracted from all the values of $\epsilon$

- Condition (ii): $50 \%$ subtracted from all the values of $d_{\|_{o}} ; 5 \%$ subtracted from all the values of $\mathrm{e}_{\exp }$ and $0.1 \%$ added to all the values of $\epsilon$

The influence of the experimental percentage errors of Conditions (i) and (ii) on the predicted permeability is shown in fig. 3. Condition (i) can be regarded as an upper bound for the permeability prediction of the anisotropic short duct foam RUC model and Condition (ii) as a lower bound. The permeability prediction provided by the anisotropic short duct RUC model is enveloped between the two bounding curves. Also shown in fig. 3 are Condition (ii) for a $25 \%$ and $5 \%$ error in $d_{\|_{o}}$.

Fig. 3 illustrates that, although the experimental error range in the value of $d_{\|_{o}}$ cannot be justified (since the hydraulic diameter is not specified) the model shows that it has the ability to yield satisfactory predictions. The model prediction takes on a similar trend as the experimental data. It is worth mentioning that in the sensitivity analysis performed in the present study, the specific error range considered for each parameter was assumed to be applicable to all the data points. It may happen that some data points were measured with more accuracy than others. Knowing this information may improve the model prediction even more.

The over-predicted values at the two highest porosities, may be attributed to the assumption of flow between parallel plates that breaks down at these high porosity values. It may also be that the fibrous porous structure cannot initially be assumed to be isotropic as it is assumed in the model.

Due to the lack of analytical models from the literature that can resemble the geometric porous micro-structure and account for the effects of compression and developing flow on permeability, Akaydin et al. [7] applied the semi-empirical model of Mirbod et al. [10] to their experimental permeability data. The model of Mirbod et al. [10] is based on a modification of the semi-empirical CarmanKozeny equation citeBird. Application of the model of Mirbod et al. [10] to the porosities presented in Table 1 will, however, not account for the effect of compression nor developing flow on permeability. It will yield the same permeability values as for an isotropic structure of the same porosity values in the absence of compression. In addition, since the model of Mirbod et al. [10] is based on an empirical relation it cannot be physically adapted to incorporate these physical phenomena to improve its predictive capabilities. 


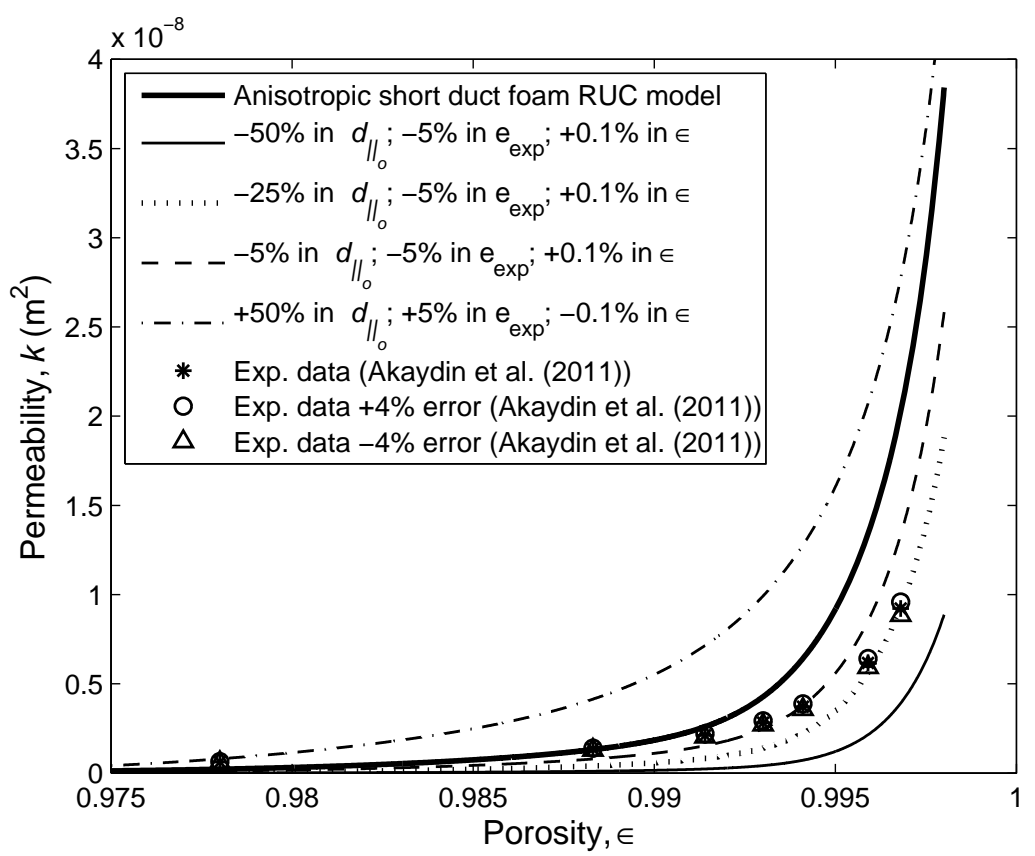

Figure 3: Influence of Condition (i) and (ii) together with various experimental error ranges in $d_{\|_{o}}, \mathrm{e}_{\exp }$ and $\epsilon$ on the permeability.

\section{Conclusions}

An adaptable geometric pore-scale model was used to predict the permeability of a soft fibrous porous medium subject to different stages of compression in the streamwise direction. It was shown that the anisotropic model decreases the over-prediction of the isotropic model by an average of $94.0 \%$. The permeability prediction of the anisotropic RUC model was then adapted to account for the effect of developing flow. It was illustrated that already at these low Reynolds numbers the effect of developing flow is significant and has to be accounted for in the permeability prediction. The incorporation of the effect of developing flow reduced the over-prediction of the anisotropic model by another $65.7 \%$, on average. The permeability ranges over more than an order of magnitude over the relatively low porosity ranges involved. A sensitivity analysis was performed on the model predictions to determine combinations of the specified experimental percentage error ranges that yield an upper bound and lower bound for the permeability prediction. The absolute relative percentage differences between the two bounding permeability curves are up to about $150 \%$. This shows that the 
anisotropic RUC model is very sensitive to deviations in the measured microstructural input parameters and has a similar trend as the experimental data.

\section{Acknowledgements}

This work is based on the research supported by the National Research Foundation. Any opinion, finding and conclusion or recommendation expressed in this material is that of the author and the NRF does not accept any liability in this regard.

\section{References}

[1] Dukhan, N. \& Patel, P., Equivalent particle diameter and length scale for pressure drop in porous metals. Experimental Thermal and Fluid Science, 32(5), pp. 1059-1067, 2008.

[2] Gerbaux, O., Vercueil, T., Memponteil, A. \& Bador, B., Experimental characterization of single and two-phase flow through nickel foams. Chemical Engineering Science, 64(19), pp. 4186-4195, 2009.

[3] Dukhan, N., Correlations for the pressure drop for flow through metal foam. Experiments in Fluids, 41(4), pp. 665-672, 2006.

[4] Antohe, B.V., Lage, J.L., Price, D.C. \& Weber, R.M., Experimental determination of permeability and inertia coefficients of mechanically compressed aluminum porous matrices. Journal of Fluids Engineering, 119(2), pp. 404-412, 1997.

[5] Boomsma, K. \& Poulikakos, D., The effects of compression and pore size variations on the liquid flow characteristics in metal foams. Journal of Fluids Engineering, 124(1), pp. 263-272, 2002.

[6] Du Plessis, J.P., Montillet, A., Comiti, J. \& Legrand, J., Pressure drop prediction for flow through high porosity metallic foams. Chemical Engineering Science, 49(21), pp. 3545-3553, 1994.

[7] Akaydin, H.D., Pierides, A., Weinbaum, S. \& Andreopoulos, Y., Permeability of soft porous media under one-dimensional compaction. Chemical Engineering Science, 66(1), pp. 1-14, 2011.

[8] Churchill, S.W. \& Usagi, R., A general expression for the correlation of rates of transfer and other phenomena. The American Institute of Chemical Engineers Journal, 18(6), pp. 1121-1128, 1972.

[9] Shah, R.K. \& London, A.L., Laminar flow forced convection in ducts. Advances in Heat Transfer, Supplement 1, Academic Press, London, United Kingdom, 1978.

[10] Mirbod, P., Andreopoulos, Y. \& Weinbaum, S., On the generation of lift forces in random soft porous media. Journal of Fluid Mechanics, 619, pp. 147-166, 2009.

[11] Bird, R.B., Stewart, W.E. \& Lightfoot, E.N., Transport Phenomena. Revised Second Edition, John Wiley \& Sons, Inc., New York, United States of America, 2007. 